\title{
Everett's pure wave mechanics and the notion of worlds
}

\author{
Jeffrey A. Barrett
}

Received: 29 August 2010 / Accepted: 29 November 2010 / Published online: 23 March 2011

(C) The Author(s) 2011. This article is published with open access at Springerlink.com

\begin{abstract}
Everett (1957a, b, 1973) relative-state formulation of quantum mechanics has often been taken to involve a metaphysical commitment to the existence of many splitting worlds each containing physical copies of observers and the objects they observe. While there was earlier talk of splitting worlds in connection with Everett, this is largely due to DeWitt's (Phys Today 23:30-35, 1970) popular presentation of the theory. While the thought of splitting worlds or parallel universes has captured the popular imagination, Everett himself favored the language of elements, branches, or relative states in describing his theory. The result is that there is no mention of splitting worlds or parallel universes in any of Everett's published work. Everett, however, did write of splitting observers and was willing to adopt the language of many worlds in conversation with people who were themselves using such language. While there is evidence that Everett was not entirely comfortable with talk of many worlds, it does not seem to have mattered much to him what language one used to describe pure wave mechanics. This was in part a result of Everett's empirical understanding of the cognitive status of his theory.
\end{abstract}

Keywords Hugh Everett III • Quantum measurement problem • Pure wave mechanics • Relative-states • Many worlds • Parallel universes • Emergent worlds • Bryce DeWitt

\footnotetext{
J. A. Barrett ( $\bowtie)$

Department of Logic and Philosophy of Science, University of California, Irvine, Irvine,

CA 92697, USA

e-mail: j.barrett@uci.edu
} 


\section{Pure wave mechanics and the language of relative states}

According to Bryce DeWitt's (1970) popular presentation of Hugh Everett III's (1957a) relative-state formulation of pure wave mechanics, accepting Everett's theory involves accepting the physical existence of many splitting worlds containing physical copies of observers and the objects they observe. ${ }^{1}$ Various versions this view, some significantly more subtle than DeWitt's initial and subsequent formulations, remain fashionable. ${ }^{2}$ Such many-worlds interpretations of quantum mechanics are often interesting in their own right, but they do not necessarily mesh well with Everett's own views.

While there is no single interpretation of Everett that fits well with all of his published work, marginal notes, and correspondence, some features of his project are clear. Everett's commitment to pure wave mechanics, described using the language of relative states, and his commitment to the goal of showing that this theory makes the same empirical predictions as the standard collapse theory remained constant throughout both his published and unpublished work. Moreover, he had much to say concerning both how he understood pure wave mechanics and how he understood physical theories generally. Together this evidence explains both why it is unlikely that Everett would have taken his theory to require or even to support the metaphysical reality of many worlds, and why it is unlikely that he would have cared much what language people used to describe his theory.

Pure wave mechanics, the theory Everett consistently championed, was for him simply the standard von Neumann-Dirac formulation of quantum mechanics without the collapse dynamics. In order to resolve the quantum measurement problem encountered by the standard collapse formulation, Everett proposed taking pure wave mechanics to be a complete physical

\footnotetext{
${ }^{1}$ DeWitt was the first to present Everett's theory in print as involving a metaphysical commitment to many worlds. There are at least two earlier mentions of worlds in connection with Everett. In the earliest, at a conference on quantum gravity organized by John Wheeler in January 1957, Cecile DeWitt reports that Richard Feynman said that "the concept of a 'universal wave function' has serious difficulties" and that "this is so since the function must contain amplitudes for all possible worlds depending upon all quantum mechanical possibilities in the past and thus one is forced to believe in the equal reality of an infinity of possible worlds" (DeWitt 1957). While this is not reported as a direct quotation, it is likely a close representation of Feynman's position at the time. The second was at the Xavier conference on the conceptual foundation of quantum mechanics discussed below.

${ }^{2}$ See Byrne (2010) for a presentation of the popular many-worlds view of Everett. See Saunders et al. (2010), and especially Wallace $(2010,2011)$, for discussions of recent decoherence formulations of many-worlds interpretations. Rather than postulate worlds as metaphysically basic, the Saunders-Wallace emergent-world interpretation of Everett takes the quantum state to be all that there is. Worlds are emergent, but real, physical entities. We will return to this idea below. In contrast, see Osnaghi et al. (2009) for a close historical reading of Everett's relative-state formulation as a challenge to the Copenhagen orthodoxy.
} 
theory. ${ }^{3}$ His goal was to "deduce the probabilistic assertions of Process 1 as subjective appearances ... thus placing the theory in correspondence with experience. We are then led to the novel situation in which the formal theory is objectively continuous and causal, while subjectively discontinuous and probabilistic" $(1973,9)$.

Everett's deductive strategy was to find a representation of our quantum mechanical experience in the correlation model described by pure wave mechanics. The first step in finding a representation of our experience was to model ideal observers in the theory. If an ideal observer $M$ begins in a ready-to-make-a-measurement state and measures the observable $O$ of system $S$, with eigenstates $\phi_{S}^{i}$, then $M$ 's memory becomes correlated to $S$ 's state:

$$
|\operatorname{ready}\rangle_{M} \sum_{i} a_{i} \phi_{S}^{i} \longrightarrow \sum_{i} a_{i}\left|a_{i}\right\rangle_{M} \phi_{S}^{i}
$$

Hence, the linearity of the dynamics in pure wave mechanics "has the far reaching implication that for any possible measurement, for which the initial system state is not an eigenstate, the resulting state of the composite system leads to no definite system state nor any definite apparatus state" $(1973,60)$.

Everett distinguished between absolute and relative states. The interpretation of absolute states was given by the standard eigenvalue-eigenstate link. This is why there is no definite system or apparatus state for any proper part of the composite subsystem described by the entangled postmeasurement above. Both the system and apparatus, however, do possess fully determinate relative states.

One can arbitrarily choose a state for one subsystem, and be led to the relative state for the remainder. Thus we are faced with a fundamental relativity of states, which is implied by the formalism of composite systems. It is meaningless to ask the absolute state of a subsystem-one can only ask the state relative to a given state of the remainder of the subsystem. (1957b, 317)

It is in relative states that Everett finds our actual quantum mechanical experience.

Given the linearity of the dynamics

[i]t is then an inescapable consequence that after the interaction has taken place there will not, generally, exist a single observer state. There will, however, be a superposition of the composite system states, each element of which contains a definite observer state and a definite relative

\footnotetext{
${ }^{3}$ See Albert (1992) and Barrett $(1999,2008)$ for a description of the measurement problem and for an explanation of the sense in which this would immediately resolve the measurement problem as faced by the standard collapse theory.
} 
object-system state. Furthermore, as we shall see, each of these relative object-system states will be, approximately, the eigenstates of the observation corresponding to the value obtained by the observer which is described by the same element of the superposition. Thus, each element of the resulting superposition describes an observer who perceived a definite and generally different result, and to whom it appears that the object-system state has been transformed into the corresponding eigenstate. In this sense the usual assertions of [the collapse of the state on measurement] appear to hold on a subjective level to each observer described by an element of the superposition. $(1973,10)$.

Particular experience, then, is explained by the fact that there is a decomposition of the universal state where one can find the particular experience of an observer; more precisely, a particular determinate experience is explained by there being a relative observer state that describes the observer as having the particular experience and by this relative state of the observer being associated with a corresponding relative state for the observed system.

It is clear that Everett proposed pure wave mechanics as a complete and accurate physical theory and that he took relative states to explain experience. The two perennial interpretational puzzles concern (1) how he understood relative states, elements, or branches and (2) how he understood probability. This paper concerns the first problem.

\section{DeWitt's splitting worlds}

DeWitt believed that Everett meant for each term in a preferred decomposition of the absolute state to describe a metaphysically real copy of the physical world. Indeed, DeWitt attributed this view to Everett, Everett's thesis advisor John Wheeler, and DeWitt's graduate student R. Neill Graham. In his popular presentation of the theory DeWitt (1970), consequently, called it the EWG interpretation, and he presented the metaphysical commitment to splitting worlds as its central feature.

DeWitt introduced the EWG metaphysical commitment to real, causally closed worlds in the context of the Schrödinger's cat thought experiment.

The animal [is] trapped in a room together with a Geiger counter and a hammer, which, upon discharge of the counter, smashes a flask of prussic acid. The counter contains a trace of radioactive material-just enough that in $1 \mathrm{~h}$ there is a $50 \%$ chance one of the nuclei will decay and therefore an equal chance the cat will be poisoned. At the end of the hour the total wave function for the system will have a form in which the living cat and the dead cat are mixed in equal portions. Schrodinger felt that the wave mechanics that led to this paradox presented an unacceptable description of reality. However, Everett, Wheeler and Graham's interpretation of 
quantum mechanics pictures the cats as inhabiting two simultaneous, noninteracting, but equally real worlds. $(1970,31)$

DeWitt took this to follow from "the mathematical formalism of quantum mechanics as it stands without adding anything to it." More specifically, DeWitt claimed that EWG had proven a metatheorem that the mathematical formalism of pure wave mechanics interprets itself.

Without drawing on any external metaphysics or mathematics other than the standard rules of logic, EWG are able, from these postulates, to prove the following metatheorem: The mathematical formalism of the quantum theory is capable of yielding its own interpretation. $(1970,33)$

DeWitt gave Everett credit for the metatheorem, Wheeler credit for encouraging Everett, and R. Neill Graham credit for clarifying the metatheorem.

Everett believed that pure wave mechanics was capable of yielding its own interpretation in that it allowed one to deduce the empirical predictions of the standard collapse theory. The question here concerns exactly how this was supposed to work. DeWitt thought that pure wave mechanics alone entailed the existence of many splitting worlds and that these worlds then explained why one should expect to the experiences predicted by the standard collapse theory. The extent to which pure wave mechanics might be taken to provide its own metaphysical interpretation, however, is subtle both historically and conceptually.

Since, as a point of logic, purely mathematical postulates entail only purely mathematical theorems, one cannot deduce any metaphysical commitments whatsoever regarding the physical world from the mathematical formalism of pure wave mechanics alone. Consequently, pure wave mechanics can only entail the sort of metaphysical commitments that DeWitt envisioned if a proper statement of the theory is already taken to involve interpretational principles that go beyond the bare mathematical formalism. The right question then is whether and to what extent pure wave mechanics might be taken to provide its own interpretation by dint of interpretational principles that one might properly understand as a natural part of the theory. That said, if DeWitt meant to suggest that Everett had proven a metatheorem that pure wave mechanics, even properly conceived, involves a commitment to the existence of metaphysically real splitting worlds, then it is unclear what he could have had in mind. On even a very broad understanding of what might count as such a metatheorem, there is nothing answering to DeWitt's description in either the long or short versions of Everett's thesis. Of course, that Everett never claimed to have deduced the existence of splitting worlds from a version of pure wave mechanics does not mean that it is impossible to do so. But whether and to what extent such a deduction might be possible depends on what metaphysical assumptions one takes to be properly included in a full statement of pure wave mechanics. 
The emergent-world interpretation of Everett as characterized by Wallace (2010) provides an example of what it would take to deduce worlds from a version of pure wave mechanics and is, hence, worth a short digression. ${ }^{4}$ While Wallace takes Everett's theory to be pure wave mechanics, his understanding of the theory involves significantly more than than just a statement of the mathematical formalism. ${ }^{5}$ Pure wave mechanics, properly conceived, is taken to postulate that at the most fundamental level "the quantum state is all there is." Further, the quantum state is to be thought of as physically real in the same sense as a field might be taken as physically real in classical field theory. Worlds on this view are to understood as emergent entities. More specifically, worlds are "mutually dynamically isolated structures instantiated within the quantum state, which are structurally and dynamically 'quasiclassical" " where the "existence of these "worlds' is established by decoherence theory" (2010, 69-70). The thought then is to identify worlds in terms of approximate emergent substructures of the correlation structure exhibited by the quantum state. Since the quantum state is physically real, worlds are also physically real inasmuch as they are identified with structures of the quantum state. It is in characterizing the emergence of worlds that the theory does some of its own interpretation work, but telling the story of emergent worlds here requires a prior commitment to an appropriate version of state realism.

In contrast with DeWitt's reading of Everett, then, Wallace does not insist that one somehow gets the metaphysics of splitting worlds from the mathematical formalism of pure wave mechanics alone. Rather, as one would expect, in order to get worlds as physically real emergent entities, one must first stipulate the more basic ontology from which they emerge. Here this means adopting a version of quantum state realism where the universal wave function represents the sort of physical entity whose decohering substructures might be properly identified as physically real emergent worlds. Rather than deriving this metaphysical commitment from the mathematical formalism, it is simply assumed as a primitive postulate of the theory, properly conceived, that the quantum state is all there is and that it is the sort of entity that might explain the existence of physically real emergent worlds by exhibiting an appropriate structure under the dynamics. The point here is that while it may be possible to argue that pure wave mechanics somehow yields its own interpretation, this would require a significantly richer understanding of the theory than one can

\footnotetext{
${ }^{4}$ While there are many people who hold some version of the emergent-world interpretation and while something like this view goes back to at least Zurek (1991) and arguably to Gell-Mann and Hartle (1990) and Zeh (1970). Simon Saunders and David Wallace have recently done much of the conceptual work required to make sense of this approach.

${ }^{5}$ Wallace takes the emergent-world interpretation to be identical with pure wave mechanics and consequently to involve no additions to the theory itself. One might better understand the emergent-world interpretation as pure wave mechanics together with decoherence considerations and a metaphysical interpretation of the theory in light of such considerations.
} 
have from the mathematical formalism alone, an understanding that already involves significant interpretational commitments. ${ }^{6}$

After claiming that Everett had proven a metatheorem that the mathematical formalism of pure wave mechanics yields an interpretation of the theory without drawing on external metaphysics, DeWitt reported that

[t]he obstacle to taking such a lofty view of things, of course, is that it forces us to believe in the reality of all the simultaneous worlds represented in the superposition [...] in each of which the measurement has yielded a different outcome. Nevertheless, this is precisely what EWG would have us believe. According to them the real universe is faithfully represented by a state vector similar to that [above] but of vastly greater complexity. This universe is constantly splitting into a stupendous number of branches, all resulting from the measurement like interactions between its myriads of components. Moreover, every quantum transition taking place on every star, in every galaxy, in every remote corner of the universe is splitting our local world on earth into myriads of copies of itself. $(1970,33)$

And concerning this vast and nonlocal splitting of physical worlds, DeWitt famously reflected that

I can recall vividly the shock I experienced on first encountering this multiworld concept. The idea of $10^{100+}$ slightly imperfect copies of oneself all constantly splitting into further copies, which ultimately become unrecognizable, is not easy to reconcile with common sense. Here is schizophrenia with a vengeance. ... Here we must surely protest. We do not split in two, let alone into $10^{100+}$ ! To this EWG reply: To the extent that we can be regarded simply as automata and hence on a par with

\footnotetext{
${ }^{6}$ The historical question of how one might best reconstruct Everett's own understanding of pure wave mechanics and its interpretation is discussed to some extent below and in more detail in Barrett (2010, 2011). There is good reason to suppose that (1) Everett's understanding of pure wave mechanics did not involve any metaphysical commitment to the physical existence of the quantum state or to real splitting worlds, (2) it was important to Everett's understanding of branches that they might be individuated with respect to any basis whatsoever and that they are never in principle dynamically isolated, and (3) Everett did not appeal to decoherence considerations to individuate branches. The emergent-worlds interpretation is nevertheless worthy of serious consideration on its own merits. Further, this relatively recent interpretation of Everett does capture at least one centrally important aspect of his project: Everett sought to demonstrate the empirical faithfulness of pure wave mechanics by showing that one could find records corresponding to the usual statistical prediction of quantum mechanics in the correlation structure characterized by the global quantum state. At some level of description, this is precisely what is done in the Saunders-Wallace interpretation; it is just that Everett did not explicitly consider this particular way of individuating quasiclassical substructures.
} 
ordinary measuring apparatuses, the laws of quantum mechanics do not allow us to feel the splits. $(1970,33)^{7}$

And, on behalf of Everett, Wheeler, and Graham, DeWitt concluded:

Finally, the EWG interpretation of quantum mechanics has an important contribution to make to the philosophy of science. By showing that formalism alone is sufficient to generate interpretation, it has breathed new life into the old idea of a direct correspondence between formalism and reality. The reality implied here is admittedly bizarre. To anyone who is awestruck by the vastness of the presently known universe, the view from where Everett, Wheeler and Graham sit is truly impressive. $(1970,35)$

When DeWitt and Graham subsequently included both the long and short versions of Everett's thesis with their own work in the 1973 Princeton University Press anthology The Many-Worlds Interpretation of Quantum Mechanics, the language of worlds stuck to Everett's interpretation of quantum mechanics. ${ }^{8}$ But while DeWitt's understanding of the theory may have agreed with that of Graham, there is good reason to suppose that it did not agree well with either Wheeler's or Everett's understanding. ${ }^{9}$ For his part, Wheeler directly attributed the many worlds interpretation of Everett to DeWitt.

Bryce DeWitt, my friend at Chapel Hill, chose to call the Everett interpretation the "many worlds" interpretation, and DeWitt's terminology is now common among physicists (although I don't like it). The idea has entered into the general public consciousness through the idea of "parallel universes." Although I have coined catchy phrases myself to try to make an idea memorable, in this case, I opted for a cautious, conservative term. "Many worlds" and "parallel universes" were more than I could swallow. (Wheeler and Ford 1998, 269-270)

\footnotetext{
${ }^{7}$ While $10^{100+}$ is a big number, DeWitt believed that there were only a finite number of worlds because of the finite precision of our measurements $(1971,42)$. For his part, Everett took there to be an uncountably infinite number of branches depending on the decomposition of the state one considers. See Werner (1962).

${ }^{8}$ Everett (1973) is a slightly revised version of the original long version of Everett's Ph.D. thesis circulated in 1956. Everett (1957a) is the much shorter official version of his thesis as revised and redacted by Everett and Wheeler to soften the direct attack on the Copenhagen interpretation. This is the version that Everett defended. Everett (1957b), included in the DeWitt and Graham (1973) anthology, is virtually identical to Everett (1957a). Each of these documents presents pure wave mechanics in the language of relative states and tells essentially the same story. The main difference is in the extent to which they criticize the quantum orthodoxy of the time.

${ }^{9}$ Everett's disagreement with Graham concerned the measure of typicality appropriate for branches. Graham's intuitions concerning typicality were a result of his understanding, with DeWitt, that Everett had in mind counting branches as worlds. As discussed below, Everett was deeply frustrated by this misunderstanding.
} 
Wheeler preferred the more conservative "relative state" language for describing Everett's theory, the language that Everett in fact used consistently throughout both the long and short versions of his thesis. The choice of language one used to describe Everett's theory was not simply a matter of convention for Wheeler. Wheeler believed that DeWitt's popular many worlds language represented an "oversimplified way" of understanding Everett's theory $(1998,269)$. As we shall see, Everett's own views were indeed rather more subtle than suggested by DeWitt's popular presentation of the theory.

\section{Why Everett did not take pure wave mechanics to involve a metaphysical commitment to worlds}

While strong metaphysical commitments were central to DeWitt's understanding of Everett, Everett did not take his theory to require or even support any special metaphysical commitments.

Everett had explained his understanding of the nature and purpose of physical theories in a letter to DeWitt some years before DeWitt wrote his popular presentation of the theory.

First, I must say a few words to clarify my conception of the nature and purpose of physical theories in general. To me, any physical theory is a logical construct (model), consisting of symbols and rules for their manipulation, some of whose elements are associated with elements of the perceived world. If this association is an isomorphism (or at least a homomorphism) we can speak of the theory as correct, or as faithful. The fundamental requirements of any theory are logical consistency and correctness in this sense.... However, there is no reason why there cannot be any number of different theories satisfying these requirements, and further (somewhat arbitrary) criteria such as usefulness, simplicity, comprehensiveness, pictorability, etc., must be resorted to in such cases. There can be no question of which theory is "true" or "real"- the best that one can do is reject those theories which are not isomorphic to sense experience. (1957d)

In the second appendix to the long version of his thesis, Everett explains further that taking a theory to be descriptive of the metaphysics of the world is a methodological mistake.

[W]hen a theory is highly successful and becomes firmly established, the model tends to become identified with "reality" itself, and the model nature of the theory becomes obscured. The rise of classical physics offers 
an excellent example of this process. The constructs of classical physics are just as much fictions of our own minds as those of any other theory [...] we simply have a great deal more confidence in them. It must be deemed a mistake, therefore, to attribute any more "reality" here than elsewhere. $(1973,134)$

\section{Everett notes further that}

[o]nce we have granted that any physical theory is essentially only a model for the world of experience, we must renounce all hope of finding anything like "the correct theory." There is nothing which prevents any number of quite distinct models from being in correspondence with experience (i.e., all "correct”). (1973, 134)

So while DeWitt took a strong metaphysical commitment to splitting worlds to be central to understanding Everett's theory, Everett believed that his theory neither required nor supported any special metaphysical commitments. As Everett explained in the long thesis, 'the primary purpose of theoretical physics is ... to make useful models which serve for a time and are replaced as they are outworn" $(1973,111){ }^{10}$

This does not mean, however, that Everett was only interested in the purely positivistic virtues of his theory. While he held that one can only require that that a physical theory be logically consistent and empirically faithful, he also believed that there were various optional, but desirable, pragmatic virtues. ${ }^{11}$ Everett described this aspect of theory selection in terms of a sort of costbenefit analysis. Among the pragmatic virtues was pictorability, and there is reason to believe that Everett took pure wave mechanics to have this virtue. ${ }^{12}$ The pictorability of a theory might be thought to involve a particularly detailed intuitive understanding of the theory's model.

\footnotetext{
${ }^{10}$ This is presumably why Everett consistently used scare quotes around language that might be interpreted as involving metaphysical commitments. Everett took his view of theories to be "very nearly identical" with the view held by the positivist Philipp Frank in the 1950's (Everett 1957c). For his part, Frank identified agreement with observations as the primary virtue of physical theories and allowed for secondary virtues such as simplicity; but since one never has perfect agreement with observations nor perfect simplicity, Frank argued, our physical theories will be, at best, in fair agreement with observations and of sufficient simplicity to be useable. Frank thus concluded that "it is obvious that such a theory cannot be "the truth"; rather, it is "an instrument that serves toward some definite purpose .... a tool that produces other tools according to a practical scheme" (Frank 1954, 14). See Barrett (2010, 2011) for extended discussions of Everett's understanding of his project and the status of physical theories.

${ }^{11}$ See Barrett (2011) for a discussion of empirical faithfulness and other virtues in Everett and for an argument that pure wave mechanics can in fact be taken as empirically faithful on Everett's arguments alone.

${ }^{12}$ Everett mentions pictorability in connection with pragmatic virtues like simplicity and comprehensiveness, which he clearly takes pure wave mechanics to have $(1973,136)$.
} 
Everett's view concerning the proper cognitive status of his theory presents a new interpretational question for understanding Everett's project. While Everett, on methodological grounds, clearly did not believe that pure wave mechanics supported or required any special metaphysical commitments, one might consider the extent to which he favored a model or picture of the theory that involved features that are well-described using the language of many worlds.

While Everett consistently used the language of relative states, elements, and branches in describing the correlation structure of pure wave mechanics, there is (1) significant evidence that he was also willing to describe the correlation structure in terms of splitting observers, (2) some evidence that he was willing to describe it in terms of many worlds, and (3) some evidence that he took both of these sorts of description to be at least somewhat misleading and hence ultimately preferred talking in terms of relative states. I will briefly review this evidence, focussing on lesser known sources. Ultimately, largely because of the metaphysical modesty he exhibits in his discussion of the proper cognitive status of physical theories, I do not believe that it mattered much to Everett what language one used to describe the correlation model characterized by pure wave mechanics. That he did not take his theory to support or to require any special metaphysical commitments helps to explain why it has proven difficult to find a single, coherent metaphysical interpretation of his formulation of quantum mechanics that meshes well with what he actually said.

\section{Evidence for and against Everett's comfort in talking of splitting observers and many worlds}

While Everett never referred to many worlds or parallel universes in anything he wrote for publication, references to splitting observers are common in his notes and in early drafts of the long thesis, and at least some of the language of splitting observers ends up in even the final version of the long thesis.

Everett's early thesis notes include such artifacts as a remarkable analogy between splitting observers and splitting amoebas. In a short paper that Everett wrote for Wheeler to show that he was making progress on his thesis Everett argues:

We have, then, a theory which is objectively causal and continuous, while at the same time subjectively probabilistic and discontinuous. It can lay claim to a certain completeness, since it applies to all systems, of whatever size, and is still capable of explaining the appearance of the macroscopic world. The price, however, is the abandonment of the concept of the uniqueness of the observer, with its somewhat disconcerting philosophical implications. 
As an analogy one can imagine an intelligent amoeba with a good memory. As time progresses the amoeba is constantly splitting, each time the resulting amoebas having the same memories as the parent. Our amoeba hence does not have a life line, but a life tree. The question of the identity or non identity of two amoebas at a later time is somewhat vague.

At any time we can consider two of them, and they will possess common memories up to a point (common parent) after which they will diverge according to their separate lives thereafter.

We can get a closer analogy if we were to take one of these intelligent amoebas, erase his past memories, and render him unconscious while he underwent fission, placing the two resulting amoebas in separate tanks, and repeating this process for all succeeding generations, so that none of them would be aware of their splitting. After a while we would have a large number of individuals, sharing some memories with one another, differing in others, each of which is completely unaware of his "other selves" and under the impression that he is a unique individual. It would be difficult indeed to convince such an amoeba of the true situation short of actually confronting him with his "other selves". The same is true if one accepts the hypothesis of the universal wave function. Each time an individual splits he is unaware of it, and any single individual is at all times unaware of his "other selves" with which he has no interaction from the time of splitting. (1956b)

At this point in Everett's paper, Wheeler wrote in his marginal comments: "This analogy seems to me quite capable of misleading readers in what is a very subtle point. Suggest omission." The analogy does not occur again. ${ }^{13}$

In another early draft document, Everett explains:

The essence of this theory is the abandonment of the concept of the uniqueness of observers, i.e. that there are individual entities, machines, people, etc., which remain single unique individuals throughout periods of time. In this theory when measurements (or in general any observations) are made on systems by "observers" (by which we mean merely other systems) the observer itself splits into a number of observers, each of which sees a definite result for the state of the system.

\footnotetext{
${ }^{13}$ In an early handwritten document, Everett lists a number of possible thesis titles including "quantum mechanics in a world of independent observers," "inclusion of all observers into the machinery of quantum mechanics," and "the multi-observer form of quantum mechanics," (1956a). While Everett was clearly willing to talk about splitting observers, there is good reason to suppose that this is not what he had in mind here. Rather, as Everett explained in his discussion of the quantum measurement problem in the long thesis, unlike the standard von Neumann-Dirac and the Copenhagen formulations, his formulation of quantum mechanics provided a consistent framework in which more than one observer could be physically modeled.
} 
Now, all of this, which is seemingly quite farfetched and contrary to our experience, is actually implied if one takes seriously the formalism of wave mechanics [without the collapse dynamics] and we shall even see that we can recover [the collapse dynamics] from this picture as a tool of practical expediency, not as a basic hypothesis. (1956c)

These two paragraphs, however, are crossed out with a note, apparently in Everett's hand, to omit them. While this way of characterizing the essence of the theory does not occur again, the language of splitting observers does make its way into the long thesis.

When discussing reversibility and irreversibility in the long thesis Everett says:

... in observation processes the state of the observer is transformed into a superposition of observer states, each element of which describes an observer who is irrevocably cut off from the remaining elements.... As soon as the observation is performed, the composite state is split into a superposition for which each element describes a different object-system state and an observer with (different) knowledge of it. $(1973,98)$

In a particularly careful description of how to understand measurement in the long thesis, however, Everett characterizes the split in terms of states splitting rather than physical observers splitting.

We note that there is no longer any independent system state or observer state, although the two have become correlated in a one-one manner. However, in each element of the superposition (2.3), $\phi_{i} \psi_{i\left[\ldots, \alpha_{i}\right]}^{O}$, the objectsystem state is a particular eigenstate of the observer, and furthermore the observer-system state describes the observer as definitely perceiving that particular system state. It is this correlation which allows one to maintain the interpretation that a measurement has been performed. $(1973,68)$

And he then adds in a footnote:

At this point we encounter a language difficulty. Whereas before the observation we had a single observer state afterwards there were a number of different states for the observer, all occurring in a superposition. Each of these separate states is a state for an observer, so that we can speak of the different observers described by the different states. On the other hand, the same physical system is involved, and from this viewpoint it is the same observer, which is in different states for different elements of the superposition (i.e., has had different experiences in the separate elements of the superposition). In this situation we shall use the singular when we wish to emphasize that a single physical system is involved, and the plural when we wish to emphasize the different experiences for the separate elements of the superposition. (e.g., "The observer performs an 
observation of the quantity $A$, after which each of the observers of the resulting superposition has perceived an eigenvalue.") $(1973,68)$.

The suggestion seems to be that there is always precisely one physical observer for whom multiple post-measurement relative states, each characterizing different relative experiences, fully obtain. One way to understand this is in terms of a branch indexical akin to time: just as a single physical system might have different states at different times, a single physical system might have different states at different branches; or, more carefully, different states relative to different specifications of the state of any other correlated system. ${ }^{14}$ On this understanding, a careful description the measurement process involves a single physical observer possessing multiple relative states.

More generally, it is significant that Everett takes the problem here to be one of what language one should use to describe the correlation structure characterized by pure wave mechanics. Describing the relationship between the long and the short theses, Everett reports on the title page of the short thesis that "[a]n earlier less condensed draft of the present work, dated January 1956, was circulated to several physicists. Their comments were helpful in the most difficult task of finding the right words to attach to the individual constructs of the present rather straightforward mathematical machinery" (1957a). That Everett understood the problem as one of choosing the best language to describe the mathematical structure of the theory meshes well with his methodological empiricism.

\section{Early worlds}

While Everett did not refer to many worlds or parallel universes anywhere in either version of his thesis, or even in his thesis notes or drafts, Everett's formulation of quantum mechanics was presented as a theory of parallel universes well before DeWitt's popular presentation of the theory. The discussion

\footnotetext{
${ }^{14}$ The analogy between temporal and branch indexicality, between a system at different times and a system at different branches, has been developed in some detail by Saunders $(1995,1996$, 1998). See also Barrett (1999). A similar approach where physical states are understood in a purely relationally way has been recently investigated by Conroy (2010). On such interpretations of pure wave mechanics, there is precisely one quantum-mechanical world, but that world has an essentially relational structure given by the correlations that determine relative states. Property attribution to physical systems, including the attribution of having recorded a particular measurement record, then is fundamentally relational. There is a similar analogy, though perhaps less apt than the analogy with time, between special relativity and pure wave mechanics that is suggested by the language of relative states: just as the appearance of a physical system is relative to one's choice of an inertial frame in special relativity, the state of a physical system is relative to the specification of a state for a correlated system in pure wave mechanics. It is worth noting, however, that Everett clearly takes this to be an issue of linguistic convention not one of metaphysics.
} 
at the 1962 conference on the foundations of quantum mechanics at Xavier University provides an early example of such talk involving Everett himself.

When Everett's theory was first mentioned at the Xavier conference, Boris Podolsky commented "Oh yes, I remember now what it is about-it's a picture about parallel times, parallel universes, and each time one gets a given result he chooses which one of the universes he belongs to, but the other universes continue to exist" (Werner 1962, Monday AM 13). After a preliminary discussion of the theory, the participants decided that they should hear from Everett himself, so he was invited to fly to Cincinnati in order to join the conference the next day. When he had the chance to present his theory, Everett explained it as follows:

The picture I have is something like this: Imagine an observer making a sequence of observations on a number of, let's say, originally identical object systems. At the end of this sequence there is a large superposition of states, each element of which contains the observer as having recorded a particular definite sequence of results of observation. I identify a single element as what we think of as an experience, but still hold that it is tenable to assert that all of the elements simultaneously coexist. In any single element of the final superposition after all these measurements, you have a state which describes the observer as having observed a quite definite and apparently random sequence of events. Of course, it's a different sequence of events in each element of the superposition. In fact, if one takes a very large series of experiments, in a certain sense one can assert that for almost all of the elements of the final superposition the frequencies of the results of measurements will be in accord with what one predicts from the ordinary picture of quantum mechanics. That is very briefly it. (Werner 1962, Tuesday AM, 18)

Podolsky then suggested that Everett change his descriptive language.

Perhaps it might be a little clearer to most people if you put it in a different way. Somehow or other we have here the parallel times or parallel worlds that science fiction likes to talk about so much. (Werner 1962, Tuesday AM, 19)

To which Everett replied:

Yes, it's a consequence of the superposition principle that each separate element of the superposition will obey the same laws independent of the presence or absence of one another. Hence, why insist on having a certain selection of one of the elements as being real and all of the others somehow mysteriously vanishing. (Werner 1962, Tuesday AM, 19)

Later in the exchange Podolsky said "It looks like we would have a nondenumerable infinity of worlds" and Everett replied "Yes" (Werner 1962, 
Tuesday AM, 20). When Abner Shimony asked whether Everett associated awareness with each term in the superposition, Everett replied "Each individual branch looks like a perfectly respectable world where definite things have happened" (Werner 1962, Tuesday AM, 22). By conference policy Everett had the chance to revise the transcription of his own comments and, while he made several minor changes, he kept his use of the term world in this last comment.

In addition to introducing Everett to a popular audience, DeWitt's (1970) popular presentation of the many-worlds interpretation helped to generate considerable academic interest in Everett's theory. It is significant that there is no record of Everett ever publicly objecting to DeWitt's presentation of his theory. Moreover, Everett remained willing to use the language of worlds in conversation with people who preferred such language. That said, when Everett revised his long thesis for publication in the DeWitt-Graham anthology, while he made a number of changes, he kept his original language of relative states throughout. Further, Everett's private correspondence indicates that he was not entirely comfortable with DeWitt's formulation of his theory and the associated language of worlds.

In the Spring of 1977, Wheeler and DeWitt invited Everett to visit the University of Texas, Austin to give a talk on his interpretation of quantum mechanics. After the talk, David Deutsch, then a graduate student studying quantum gravity with Wheeler and DeWitt, had a conversation with Everett over lunch. DeWitt had introduced Deutsch to Everett's theory, and Deutsch took advantage of the chance to ask Everett about many universes. In an interview with the journalist Peter Byrne, Deutsch recalls Everett as being "very enthusiastic about many universes, and very robust as well as subtle in its defense, and he did not speak in terms of 'relative states' or any other euphemism" (Byrne 2010, 321-2). Deutsch has since become one of the strongest proponents of what he calls the multiverse theory, a version of DeWitt's splitting worlds interpretation of Everett. Indicating his degree of support for the theory, Deutsch claims that "[t]he fruitfulness of the multiverse theory in contributing to the solution of long-standing philosophical problems is so great that it would be worth adopting even if there were no physical evidence for it at all" $(1997,339) .{ }^{15}$

In private correspondence, Everett was explicitly asked about DeWitt's presentation of his theory on at least two occasions. This was, of course, a natural thing to ask about given the manifest differences between Everett's and DeWitt's presentations of the theory.

\footnotetext{
${ }^{15}$ As evidence for this claim, Deutsch reports that "[i]ndeed, the philosopher David Lewis, in his book On the Plurality of Worlds, has postulated the existence of a multiverse for philosophical reasons alone" (1997, 329-340). The worlds of Deutsch's quantum multiverse are, however, entirely unsuitable as a model for Lewis' modal realism. See Skyrms (1976) for an early discussion of this point.
} 
In June 1977 William Harvey, associated with the Science Studies Unit at the University of Edinburgh, wrote Everett:

The theory which you proposed in 1957 has now received widespread attention, although for ten years it was, to quote Professor Jammer, 'one of the best kept secrets in this century.' However, there are certain aspects of the revival of this fascinating theory which interest me as a historian of science.... Bryce DeWitt played a major part in the revival. Did he make contact with you, e.g. to ask your permission to publish the book he edited with Graham? After all, you had a prior claim on this theory. Do you approve of the way DeWitt presented your theory? (Harvey 1977)

Everett replied by describing the process that led to his paper being published in the DeWitt-Graham anthology; he then concludes "... I certainly approve of the way Bryce DeWitt presented my theory, since without his efforts it would never have been presented at all" (1977a).

In August of the same year, Everett was asked by the physicist JeanMarc Levy-Leblond for comments on a paper. ${ }^{16}$ Lévy-Leblond's philosophical paper was original, free-wheeling, but also well-argued for the time. In it he considered the conceptual structure of the quantum measurement problem, the Copenhagen interpretation, and finally Everett's formulation of quantum mechanics. Concerning Everett, the two main conclusions of the paper are (1) Everett believed that the central problem with the Copenhagen interpretation is that it relies too much on classical intuition and thus sacrifices a quantum understanding of the measurement process and (2) DeWitt's manyworld interpretation distorts Everett's theory by imposing classical worlds on the theory and hence making the same mistake as the Copenhagen interpretation. ${ }^{17}$ Lévy-Leblond argues that there has been a "serious misunderstanding of Everett's thesis by many of his followers." Describing the labors of Everett's propagandists:

Once more, under a question of terminology lies a deep conceptual problem. [Everett's] interpretation in effect has been called by several people, especially DeWitt, one of his main propagandists, the "manyworlds (or many-universes) interpretation of quantum theory." The rejection of the postulate projection [sic] leaves us with the "universal" state vector. Since, with each successive measurement, this state-vector "splits" into a superposition of several "branches", it is said to describe "many universes," one for each of these branches. Where the Copenhagen interpretation would arbitrarily choose "one world" by cutting off all "branches" of the state-vector except one (presumably the one

\footnotetext{
${ }^{16}$ The date on Lévy-Leblond's letter is 17 August 1978, but it is likely that he got this wrong as Everett has the date of his reply as 15 November 1977.

${ }^{17}$ The first point in fact meshes well with Everett's main argument against the Copenhagen interpretation both in his thesis and in earlier correspondence.
} 
we think we sit upon), one should accept the simultaneous existence of the "many worlds" corresponding to all possible outcomes of the measurement. Now, my criticism here is exactly symmetrical of the one I directed again the orthodox position: the "many worlds" idea again is a left-over of classical conceptions. The coexisting branches here, as the unique surviving one in the Copenhagen point of view, can only be related to "worlds" described by classical physics. The difference is that, instead of interpreting the quantum "plus" as a classical "or", DeWitt and al. interpret it as a classical "and". To me, the deep meaning of Everett's ideas is not the coexistence of many worlds, but on the contrary, the existence of a single quantum one. The main drawback of the "many-worlds" terminology is that it leads one to ask the question of "what branch we are on," since it certainly looks as if our consciousness definitely belonged to only one world at a time: But this question only makes sense from a classical point of view, once more. (1976, 184-5).

Lévy-Leblond then asked Everett whether he got it right that there are no splitting worlds.

I directly ask your opinion on what I take to be a crucial question concerning the "Everett and no-longer-Wheeler" (if I understand correctly!) interpretation of [quantum mechanics]. The question is one of terminology: to my opinion there is but a single (quantum) world, with its universal wave function. There are not "many worlds," no "branching." etc. except as an artifact of insisting once more on a classical picture of the world. (Lévy-Leblond 1978)

In reply, Everett reported that he very much liked the paper, he agreed with Lévy-Leblond's analysis of the relationship between his work and DeWitt's, and he denied that the many worlds terminology was his. Everett wrote:

The reason for the delay in acknowledging receipt of your pre-print, "Toward a Proper Quantum Theory," is that it is one of the more meaningful papers I have seen on this subject, and therefore deserving of a reply. This is always a mistake for me to make, as I very rarely complete a thorough review of papers, despite all good intentions. In this case, your observations seem entirely accurate (as far as I have read.) I especially enjoyed your inspired conclusion, pp. 192-4. ${ }^{18}$

I have not done further work in this area since the original paper in 1955. (not published in its entirety until 1973, as the "Many-Worlds Interpretation etc."). This, of course, was not my title as I was pleased to

\footnotetext{
${ }^{18}$ The inspired conclusion is Lévy-Leblond's "zipperdynamics" spoof of explanation in the Copenhagen tradition.
} 
have the paper published in any form anyone chose to do it in! I, in effect, had washed my hands of the whole affair in 1956. (1977b)

In a draft of the same letter, Everett said more about how he understood his relationship to DeWitt. Referring to DeWitt's energetic promotion of his work, Everett had it this way:

[T] he "Many-Worlds Interpretation etc." ... is not my title as I was pleased to have the paper published in any form anyone chose to do it in! I, in effect, had washed my hands of the whole affair in 1955. Far be it for me to look a gift Boswellian writer in the mouth! But your observations are entirely accurate (as far as I have read). (1977b draft) ${ }^{19}$

In agreeing with Lévy-Leblond, Everett is agreeing that the notion of many worlds relies on a mistaken classical crutch and that his own formulation of quantum mechanics is best understood in terms of a single quantum world. ${ }^{20}$ What Everett says in his draft letter also fits well with his report to Harvey that he had no objection to DeWitt's presentation of the theory since without DeWitt the theory would never have been presented at all. As Everett was not gifted at self promotion, his debt to DeWitt's Boswellian efforts was significant and he knew it. ${ }^{21}$ But he was also clearly not entirely comfortable with DeWitt's many worlds.

Everett's mentions "the world view" one last time in a letter to a physics enthusiast, L. David Raub, in 1980, two years before Everett's death. Everett begins his reply to Raub by stating that he still supports all of the conclusions of his thesis and believes that his approach remains the only entirely consistent interpretation of quantum mechanics that explains both the content of the theory and appearances. Explaining why some do not like his theory, Everett says, "It is abhorrent to many individuals that there should not be a single unique state for them (in the world view), even though my interpretation explains all subjective feelings quite adequately and is consistent with all observations" (1980). Here Everett uses both the relative state terminology of his thesis and mentions the world view. As usual, Everett suggests that what should ultimately matter is that his view is logically consistent and consistent with our observations.

\footnotetext{
${ }^{19}$ Concerning how far Everett read, he clearly read the section involving his own formulation of quantum mechanics and DeWitt's interpretation of it as a many worlds theory as he explicitly responded to material in this section. Everett's notes on his copy of the paper and what he says in his letter indicate that he also closely read at least the conclusion and the zipperdynamics sections. The earlier date in the draft letter is Everett's.

${ }^{20}$ Presumably, a single quantum world where the physical objects typically possess only relative states.

${ }^{21}$ James Boswell (1740-1795) chronicled the life and views of his friend Samuel Johnson in such a compelling way that he virtually guaranteed Johnson's enduring fame. His biography of Johnson is also famous for taking liberties with the details of Johnson's life for the purpose of engaging narrative.
} 


\section{The tension between metaphysically distinct worlds and pure wave mechanics, and the sense in which all branches are equally real for Everett}

DeWitt identified branches with metaphysically real copies of the physical worlds because this provided an immediate and compelling explanation for the existence of determinate measurement records. When a measurement occurs, the world splits, creating physical copies of the observer. Each physical copy gets a different but fully determinate measurement record that corresponds to the determinate properties of the physical objects in his world. But there is also significant tension between talk of physically splitting worlds and Everett's understanding of pure wave mechanics. One of the conflicts arises from how Everett understood the elements or branches of a superposition.

While Everett was metaphysically modest on methodological grounds, he took elements or branches to be equally real or actual both from the perspective of pure wave mechanics and in a more robust empirical sense. Everett repeatedly made the point that all branches are equally real from the perspective of the theory in that pure wave mechanics treats them in precisely the same way: both in his thesis and in his 1957 letter to DeWitt, for example, Everett reported that "[f]rom the viewpoint of the theory, all elements of a superposition (all 'branches') are 'actual,' none any more 'real' than another" (1957d). The argument, as Everett explained in his Xavier comments and elsewhere, was that since there is nothing in the theory that picks one term in the superposition as in any sense special (the fundamental relativity of states) and since, by the linearity of the dynamics, each element of the superposition obeys the same dynamical laws independent of the presence or absence of any another, the theory itself does nothing whatsoever to indicate that any particular element or branch is or need be descriptively privileged (Werner 1962, Tuesday AM, 19). But Everett also understood branches to be real in a more robust empirical sense. Specifically, because of the linearity of the dynamics,

[i]t is therefore improper to attribute any less validity or "reality" to any element of a superposition than any other element, due to [the] ever present possibility of obtaining interference effects between the elements. All elements of a superposition must be regarded as simultaneously existing. $(1973,107)$

That branches were real for Everett insofar as they might always have direct empirical consequences provides a more subtle and complete understanding of how Everett thought of branches, but it also suggests a tension between Everett's branches and DeWitt's worlds. DeWitt's conception of simultaneous, noninteracting, but equally real worlds, is not only incompatible with pure wave mechanics but it undermines Everett's positive argument for the empirical reality of branches. Further, inasmuch as both Everett's understanding 
of branches and pure wave mechanics itself require that there always be the possibility of interference between branches, branches are not so clearly worldlike. $^{22}$

Another difficulty in taking branches to be worlds in pure wave mechanics is that, in his principle of the fundamental relativity of states, Everett explicitly denies that that there is any physically preferred way in pure wave mechanics to decompose the absolute state of a physical system. Rather, one arbitrarily chooses a subsystem of the composite system and arbitrarily chooses a state for that subsystem, then the theory determines a relative state for the complement system $(1957 b, 317 ; 1973,43)$. There is hence no physically preferred way to individuate branches, and hence no physically preferred way to individuate worlds insofar as branches are understood as worlds.

In his marginal notes on a copy of the paper, Everett replies to John Bell's (1971) discussion concerning the temporal directionality of branching by insisting that the process of "branching [is] only relative to choice of basis-can make [temporal directionality of branching] either way!" (Everett's marginal notes on Bell 1971, 16). The thought is that since pure wave mechanics indicates no preferred basis, whatever one says about the branching process is relative to a choice of basis. Of course, insofar as branching is relative to a choice of basis, if one insists on associating branches with worlds then, what worlds there are is relative to a choice of basis. But if there is no canonical way

\footnotetext{
${ }^{22}$ While Everett required that branches always be capable of interference, he also recognized that, insofar as the evolution of the absolute state was linear, one could think of each branch as evolving on its own subject to the same dynamical laws, then get the resulting absolute state by superposing the independent branch evolutions by appeal to what he referred to as the superposition principle. On this view, branches might be thought of as causally independent exactly insofar as they do not in fact interfere with each other. Had it been important to Everett that branches exhibit a strong worldlike causal independence, he might have appealed to decoherence considerations to argue that branches describing sufficiently complex systems or systems that strongly correlate to their environments should never be expected to interfere. But he did not so argue in either version of his thesis. Indeed, rather than appeal to decoherence considerations to argue that branches are independent, Everett understood branches to be empirically real precisely because they always might, at least in principle, interfere with each other. Further, Everett's account of determinate measurement records relied on the postmeasurement relative states of the observer and her object system, and his account of the dynamical classicality of everyday systems relied on the low dispersion of massive systems in both position and momentum, not decoherence considerations (1973, 88-9 and 117-8; see also Barrett 2011). Given his view of worlds as causally independent, it is curious that DeWitt also, at least sometimes, seems to have understood that branches must be capable of interfering with each other and hence exhibiting unworldlike behavior: "We may, in principle, restore the interference effects ... by bringing the apparatus packets back together again. But then the correlations between system and apparatus are destroyed, the apparatus memory is wiped out and no measurement results" (1970, 35). See Albert and Barrett (1995) for further discussion of why pure wave mechanics requires interactions between branches. Regarding the emergent-worlds interpretation of Everett, there is nothing wrong with using decoherence considerations to select some branches as corresponding to emergent worlds at a specified level of description. Such emergent worlds, however, can only be thought of as more or less wellindividuated relative to the explanatory purpose at hand; put another way, not only are such worlds emergent, but how many and what emergent worlds there are is contingent on the level of description one adopts. This point is recognized in careful presentations of the view.
} 
to individuate worlds, then there are no canonical structures in the correlation model to call worlds. Of course, one could take every relative state to describe the state of a system in some world, but then there would be a different full set of equally actual worlds for every choice of basis. This would be a manymany-worlds interpretation of the correlation model, which is presumably not what DeWitt had in mind. Moreover, many of these worlds would be entirely unworldlike insofar as they would routinely exhibit interference effects. Or one might take various branches to be more or less worldlike for the explanatory purpose at hand. This would be to take the precise metaphysical distinction between different worlds to be largely a matter of convention. ${ }^{23}$

In the same paper Bell, for his part, noted the distinction between Everett and DeWitt regarding how to divide the wave function into branches and consequently on the existence of any physically preferred basis.

It is not clear to me that Everett and DeWitt conceive in the same way the division of the wave function into 'branches'. For DeWitt this division seems to be rather definite, involving a specific (although not very clearly specified) choice of variables (instrument readings) to have definite values in each branch. This choice is in no way dictated by the wave function itself (and it is only after it is made that the wave function becomes a complete description of DeWitt's physical reality). Everett on the other hand (at least in some passages) seems to insist on the significance of assigning an arbitrarily chosen state to an arbitrarily chosen subsystem and evaluating the 'relative state' of the remainder. (Bell 1971, 19)

In his personal copy of Bell's paper, Everett put a check mark suggesting agreement in the left margin next to the last sentence of this passage. ${ }^{24}$ In agreeing with Bell, Everett would simply be reiterating the view expressed in his earlier marginal notes on Bell's paper and in both versions of his thesis that the individuation of branches is arbitrary. The upshot, again, is that if one takes branches to be worlds, then the individuation of worlds is arbitrary.

Finally, if branches are supposed to describe physical copies of worlds, then one might naturally expect, as both DeWitt and Graham did, that something would be a typical property of a world if it held for most worlds. Everett's measure of typicality, however, was entirely unrelated to the number of DeWitt worlds. Moreover, Everett considered Graham's proposal to associate the typicality measure with the number of DeWitt worlds to be entirely

\footnotetext{
${ }^{23}$ I take this to be the strategy employed by the Saunders-Wallace emergent-world interpretation of Everett.

${ }^{24}$ Everett typically marked disagreement with one or more question marks, often combined with exclamation points and corrective comments. He disagreed with much of what Bell said in the Everett(?) section of this paper, but not this point. It is unclear when Everett made his marginal notes.
} 
misguided. ${ }^{25}$ Rather, Everett explicitly wanted a standard of typicality that was independent of how one chose to decompose the absolute universal state and hence independent of the number of branches one might individuate. ${ }^{26}$

The point here is that for Everett branches of the absolute state were always both conventional in their specification and empirically real in their potential to exhibit interference phenomena. One might expect any manyworlds formulation that is attributed to Everett to take into account this understanding of branches.

\section{Discussion}

As Everett reported to Lévy-Leblond, the language of many worlds was not his own. Even so, Everett was willing to use such language to describe the correlation structure characterized by pure wave mechanics when prompted to do so by Podolsky, DeWitt, and others. As a methodological empiricist, however, Everett took such talk neither to support nor to require any special metaphysical commitments. Rather, what mattered to Everett was the empirical faithfulness of pure wave mechanics, which for him involved showing how one might find our actual experience represented in the correlation structure described by the theory.

Everett took elements, branches, and relative states to be equally real both (1) from the viewpoint of the theory in the weak sense that pure wave mechanics did not distinguish between branches or between alternative decompositions of the absolute state into branches and (2) in the more robust empirical sense that pure wave mechanics required that branches always be in principle observable by virtue of potential interference effects. In other words, precisely what made branches real for Everett was their unworldlike potential to exhibit interference phenomena.

In summary, then, while Everett clearly favored the language of relative states and elements or branches in describing the correlation structure, he did not care very much what language one used to describe the structure characterized by pure wave mechanics. And while there is evidence that

\footnotetext{
${ }^{25}$ Graham thought that he was providing much needed "physical significance" to Everett's measure of typicality by associating it with the numerical proportion of DeWitt worlds for which the standard quantum statistics obtained (Graham 1970). In the margin of DeWitt's (1971) working paper where he explains how he and Graham have provided an improved understanding of the physical significance of Everett's measure of typicality, however, Everett writes "Goddam it" and adds "You don't see it."

${ }^{26}$ Everett's additivity requirement relates typicality measures under different decompositions of the state $(1973,71-72)$. If there were any single physically preferred decomposition of the state that individuated worlds, this requirement would be unmotivated. It is worth noting that Everett's additivity constraint on the typicality measure remains well-motivated on the Saunders-Wallace interpretation precisely because there is no physical matter of fact about how many worlds there are since the individuation of worlds is always contingent on the level of description one chooses.
} 
Everett was at least somewhat uncomfortable with DeWitt's description of his project and while he specifically makes a point of indicating that the talk of many worlds is not his own, he was clearly grateful to DeWitt for promoting his work.

Everett always maintained that his relative-state formulation of pure wave mechanics was both complete and fully acceptable as he had described it in his thesis. As he explained in the second appendix to the long version of the thesis, Everett took pure wave mechanics to be acceptable because it was consistent and empirical faithful, and it was superior to other consistent interpretations of quantum mechanics because it was simpler and more comprehensive. Pure wave mechanics as he presented it is indeed consistent, it is arguably simple, and it is comprehensive in the sense that all physical systems are treated in precisely the same linear way. That pure wave mechanics is empirically faithful, however, is more subtle.

While a full account goes beyond the scope of the present paper, a physical theory was empirically faithful for Everett if it provided a representation of our actual experience within a substructure of the model characterized by the theory. More specifically, Everett took pure wave mechanics to be empirically faithful because (1) the values of our measurement records are represented as relative records in the correlation structure characterized by the theory and (2) there is a measure of typicality over relative measurement records that can be determined from the correlation structure alone and that covaries with the standard quantum expectations. ${ }^{27}$

Everett recognized that there was also excess structure represented by pure wave mechanics insofar as it provided representations of both our actual measurement records and measurement records that do not appear to us to obtain, but this did not bother him since he simply required that one can be able to find our actual experience in the model and that one to be able to understand this experience as being appropriately typical. In this sense, then, Everett explained both determinate measurement records and the standard quantum statistics while avoiding the embarrassment of having to insist on a particular metaphysical interpretation of branches.

On this view, the remaining question is whether pure wave mechanics being empirically faithful in the sense described by Everett should be taken as sufficient for the empirical acceptability of the theory. While Everett's standard of empirical acceptability is certainly weaker than one might want, it is perhaps more than one might have thought one could get from pure wave mechanics without any special metaphysical commitments. Further, one might take the charitable view that the flexibility in the language that Everett used to describe the correlation model of pure wave mechanics was warranted given that he was metaphysically agnostic on epistemological grounds and that he explicitly recognized that the ordinary descriptive language did not fit well with the structure of relative states and branches in the theory. Indeed, insofar

\footnotetext{
${ }^{27}$ See Barrett $(2010,2011)$ for discussions of the empirical faithfulness of pure wave mechanics.
} 
as one insists on ordinary language explanations of experience in the context of pure wave mechanics, one will be required to use ordinary language in extraordinary ways.

Acknowledgements I would like to thank Brett Bevers, Peter Byrne, and Brian Skyrms for helpful discussions and Jim Weatherall, Thomas Barrett, David Wallace Carl Hoefer, and an anonymous referee for helpful comments on an earlier draft of this paper. This paper was funded in part by National Science Foundation Award No. SES-0924135.

Open Access This article is distributed under the terms of the Creative Commons Attribution Noncommercial License which permits any noncommercial use, distribution, and reproduction in any medium, provided the original author(s) and source are credited.

\section{References}

Albert, D. Z. (1992). Quantum mechanics and experience. Cambridge: Harvard University Press.

Albert, D. Z., \& Barrett, J. A. (1995). On what it takes to be a world. Topoi, 14(1), 35-37.

Barrett, J. A. (1999). The quantum mechanics of minds and worlds. Oxford: Oxford University Press.

Barrett, J. A. (2008). Everett's relative-state formulation of quantum mechanics. In E. N. Zalta (Ed.), The Stanford encyclopedia of philosophy (Winter 2008 Edition). URL: http://plato.stanford.edu/archives/win2008/entries/qm-everett/.

Barrett, J. A. (2010). A structural interpretation of pure wave mechanics. Humana. Mente, 13, 225 235.

Barrett, J. A. (2011). On the faithful interpretation of pure wave mechanics. British Journal for the Philosophy of Science (forthcoming).

Bell, J. (1971). On the hypothesis that the Schrödinger equation is exact. Ref.TH.1424-CERN (27 October 1971). Everett's personal copy of Bell's paper contains Everett's unpublished, undated marginal notes.

Byrne, P. (2010). The many worlds of Hugh Everett III: Multiple universes, mutual assured destruction, and the meltdown of a nuclear family. Oxford: Oxford University Press.

Conroy, C. (2010). A relative facts interpretation of everettian quantum mechanics. Ph.D. Dissertation, University of California, Irvine.

Deutsch, D. (1997). The fabric of reality: The science of parallel universes and its implications New York: Allen Lane.

DeWitt, B. S. (1970). Quantum mechanics and reality. Physics Today, 23, 30-35.

DeWitt, B. S. (1971). The many universes interpretation of quantum mechanics. Preprint. Department of Physics University of North Carolina at Chapel Hill. Reprinted in DeWitt and Graham (1973). Everett's personal copy has two significant marginal notes.

DeWitt, B. S., \& Neill Graham, R. (eds.) (1973). The many-worlds interpretation of quantum mechanics. Princeton: Princeton University Press.

DeWitt, C. M. (1957). Conference on the role of gravitation in physics. Transcript. National Technical Information Service, AD118180.

Everett, H., III (1956a). Handwritten notes on possible thesis titles. Undated single half page manuscript in pencil, blue pen, and red pencil. In the thesis drafts (handwritten), notes and misc file. Forthcoming in the UCISpace Everett archive funded by NSF Award No. SES0924135.

Everett, H., III (1956b). Probability in wave mechanics. Undated nine page typescript addressed to John Wheeler with Wheeler's marginal notes. In thesis drafts, mini-papers file. Forthcoming in the UCISpace Everett archive funded by NSF Award No. SES-0924135.

Everett, H., III (1956c). Origins of the probabilistic aspects of quantum mechanics. Undated seven page typescript with Everett's marginal notes and corrections. In thesis materials file. Forthcoming in the UCISpace Everett archive funded by NSF Award No. SES-0924135.

Everett, H., III (1957a). On the foundations of quantum mechanics. Ph.D. thesis, Princeton University, Department of Physics. 
Everett, H., III (1957b). Relative state formulation of quantum mechanics. Reviews of Modern Physics, 29, 454-462. (Reprinted in DeWitt and Graham (1973) and in Wheeler and Zurek (1983)).

Everett, H., III (1957c). Letter from Hugh Everett III to Philipp G. Frank dated May 31, 1957.

Everett, H., III (1957d). Letter from Hugh Everett III to Bryce DeWitt dated May 31, 1957. Forthcoming in the UCISpace Everett archive funded by NSF Award No. SES-0924135.

Everett, H., III (1973). The theory of the universal wave function. In B. S. DeWitt, \& N. Graham (Eds.), The many-worlds interpretation of quantum mechanics (pp. 3-140). Princeton University Press.

Everett, H., III (1977a). Letter from Hugh Everett, III to William Harvey dated June 20, 1977.

Everett, H., III (1977b). Letter from Hugh Everett III to Jean-Marc Lévy-Leblond dated November 15, 1977. Everett filed a copy of this letter with his draft. The draft contains Everett's corrections and marginal notes. Forthcoming in the UCISpace Everett archive funded by NSF Award No. SES-0924135.

Everett, H., III (1980). Letter from Hugh Everett, III to L. David Raub dated April 7, 1980.

Frank, P. (1954). The validation of scientific theories. Boston: Beacon Press.

Gell-Mann, M., \& Hartle, J. B. (1990). Quantum mechanics in the light of quantum cosmology. In W. H. Zurek (Ed)., Complexity, entropy, and the physics of information. Proceedings of the Santa Fe institute studies in the sciences of complexity (Vol. VIII, pp. 425-458). Redwood City: Addison-Wesley.

Graham, N. (1970). The Everett interpretation of quantum mechanics. Ph.D. Dissertation, University of Noth Carolina at Chapel Hill.

Harvey, W. (1977). Letter from William Harvey to Hugh Everett, III dated June 8, 1977.

Lévy-Leblond, J.-M. (1976). Towards a proper quantum theory (hints for a recasting). Dialectica, $30(2 / 3), 162-196$.

Lévy-Leblond, J.-M. (1978). Letter from Jean-Marc Lévy-Leblond to Hugh Everett III dated August 17, 1978 but likely written August 17, 1977 since Everett's reply is dated November $15,1977$.

Osnaghi, S., Freitas, F., \& Freire, O. (2009). The origin of the Everettian Heresy. Studies in History and Philosophy of Modern Physics, 40, 97-123. doi:10.1016/j.shpsb.2008.10.002.

Saunders, S. (1995). Time, quantum mechanics, and decoherence. Synthese, 102, 235-266.

Saunders, S. (1996). Time, quantum mechanics, and tense. Synthese, 107, 19-53.

Saunders, S. (1998). Time, quantum mechanics, and probability. Synthese, 114, 373-404.

Saunders, S., Barrett, J., Kent, A., \& Wallace, D. (Eds) (2010). Many worlds? Everett, quantum theory, and reality. Oxford: Oxford University Press.

Skyrms, B. (1976). Possible worlds, physics and metaphysics. Philosophical Studies, 30(5), 323-332.

Wallace, D. (2010). Decoherence and ontology. In Saunders, et al. (Eds) (pp. 53-72).

Wallace, D. (2011). The emergent multiverse: Quantum theory according to the Everett interpretation. Oxford University Press (Forthcoming).

Werner, F. G. (ed.) (1962). Proceedings of the conference on the foundations of quantum mechanics, Xavier University, Cincinnati, Ohio; October 1-5, 1962. Conference Chairman Boris Podolsky. Conference reporter Frederick G. Werner. Transferred to cd in June 2002 for the Xavier University Department of Physics by Joseph G. Brinkman.

Wheeler, J., \& Ford, K. (1998). Geons, black holes, and quantum foam. New York: Norton.

Zeh, H. D. (1970). On the interpretation of measurement in quantum theory. Foundations of Physics, 1, 69-76.

Zurek, W. H. (1991). Decoherence and the transition from quantum to classical. Physics Today, 44(10), 36-44. 